\section{( OPEN ACCESS}

\title{
Treatment of psoriatic arthritis in a phase 3 randomised, placebo-controlled trial with apremilast, an oral phosphodiesterase 4 inhibitor
}

\author{
Arthur Kavanaugh, ${ }^{1}$ Philip J Mease, ${ }^{2}$ Juan J Gomez-Reino, ${ }^{3}$ Adewale 0 Adebajo, ${ }^{4}$ \\ Jürgen Wollenhaupt, ${ }_{1}^{5}$ Dafna D Gladman, ${ }^{6}$ Eric Lespessailles, ${ }^{7}$ Stephen Hall, ${ }^{8}$ \\ Marla Hochfeld, ${ }^{9}$ ChiaChi Hu, ${ }^{9}$ Douglas Hough, ${ }^{9}$ Randall M Stevens, ${ }^{9}$ Georg Schett ${ }^{10}$
}

\begin{abstract}
Handling editor Tore K Kvien
- Additional material is published online only. To view please visit the journal online (http://dx.doi.org/10.1136/ annrheumdis-2013-205056).
\end{abstract}

For numbered affiliations see end of article.

\section{Correspondence to}

Dr Arthur Kavanaugh, Division of Rheumatology, Allergy and Immunology, Department of Medicine, University of California, San Diego 9500 Gilman Drive, Mail Code 0943, La Jolla, CA 92093-0943, USA; akavanaugh@ucsd.edu

Received 11 December 2013 Revised 11 February 2014 Accepted 16 February 2014 Published Online First 4 March 2014

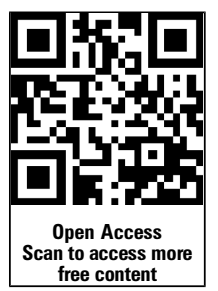

To cite: Kavanaugh $A$ Mease PJ, Gomez-Reino JJ, et al. Ann Rheum Dis 2014;73:1020-1026.

\section{ABSTRACT}

Objectives Apremilast, an oral phosphodiesterase 4 inhibitor, regulates inflammatory mediators. Psoriatic Arthritis Long-term Assessment of Clinical Efficacy 1 (PALACE 1) compared apremilast with placebo in patients with active psoriatic arthritis despite prior traditional disease-modifying antirheumatic drug (DMARD) and/or biologic therapy.

Methods In the 24-week, placebo-controlled phase of PALACE 1, patients $(N=504)$ were randomised $(1: 1: 1)$ to placebo, apremilast $20 \mathrm{mg}$ twice a day (BID) or apremilast $30 \mathrm{mg}$ BID. At week 16, patients without $\geq 20 \%$ reduction in swollen and tender joint counts were required to be re-randomised equally to either apremilast dose if initially randomised to placebo or remained on their initial apremilast dose. Patients on background concurrent DMARDs continued stable doses (methotrexate, leflunomide and/or sulfasalazine). Primary outcome was the proportion of patients achieving $20 \%$ improvement in modified American College of Rheumatology response criteria (ACR20) at week 16. Results At week 16, significantly more apremilast $20 \mathrm{mg} \mathrm{BID} \mathrm{(31 \% )} \mathrm{and} 30 \mathrm{mg}$ BID (40\%) patients achieved ACR20 versus placebo (19\%) $(p<0.001)$. Significant improvements in key secondary measures (physical function, psoriasis) were evident with both apremilast doses versus placebo. Across outcome measures, the 30-mg group generally had higher and more consistent response rates, although statistical comparison was not conducted. The most common adverse events were gastrointestinal and generally occurred early, were self-limiting and infrequently led to discontinuation. No imbalance in major adverse cardiac events, serious or opportunistic infections, malignancies or laboratory abnormalities was observed.

Conclusions Apremilast was effective in the treatment of psoriatic arthritis, improving signs and symptoms and physical function. Apremilast demonstrated an acceptable safety profile and was generally well tolerated.

Clinical trial registration number NCT01172938.

\section{INTRODUCTION}

Psoriatic arthritis (PsA) is an inflammatory arthritis present in up to $30 \%$ of patients with psoriasis, with an estimated prevalence of $0.3-1.0 \%$ of the general population. ${ }^{1}$ While there are therapeutic options for PsA, not all patients respond to or tolerate currently available treatments. ${ }^{2-5}$ Thus, a need exists for additional oral treatment options with a novel mechanism of action.

Apremilast is an oral phosphodiesterase 4 inhibitor that has been shown to regulate inflammatory mediators. ${ }^{6}$ Phosphodiesterase 4 , the dominant phosphodiesterase expressed in immune cells, degrades cyclic AMP (cAMP) into AMP. Phosphodiesterase 4 inhibition thereby elevates intracellular cAMP, which can down-regulate the inflammatory responses through mechanisms such as partially inhibiting expression of inflammatory cytokines and increasing expression of anti-inflammatory mediators such as interleukin-10. ${ }^{67}$

The phase 3 Psoriatic Arthritis Long-term Assessment of Clinical Efficacy (PALACE) clinical trial programme assessed the efficacy, tolerability and safety of apremilast in patients with active PsA across four placebo-controlled trials. This report describes results from the first trial (PALACE 1) that enrolled patients with active PsA despite prior traditional disease-modifying antirheumatic drugs (DMARDs) and/or biologics.

\section{METHODS \\ Patients}

Patients were eligible for enrolment if they were $\geq 18$ years of age and were diagnosed with active PsA. The institutional review boards at each participating medical centre approved the protocol and all patients provided written informed consent before study entry. Patients were required to meet the Classification Criteria for Psoriatic Arthritis $(\mathrm{CASPAR})^{8}$ at screening and have a minimum of both three swollen and three tender joints, despite prior treatment with traditional DMARDs and/or biologic treatment or concurrent treatment with traditional DMARDs. Prior tumour necrosis factor blocker efficacy failures were limited to $\leq 10 \%$ of enrolled patients. Patients taking methotrexate, leflunomide or sulfasalazine must have been treated for at least 16 weeks and on a stable dose (oral or parenteral methotrexate $\leq 25 \mathrm{mg} /$ week; leflunomide $\leq 20 \mathrm{mg} /$ day; sulfasalazine $\leq 2 \mathrm{~g} /$ day; or a combination) for at least 4 weeks before the screening visit. Stable doses of oral corticosteroids (prednisone $\leq 10 \mathrm{mg} /$ day or equivalent for at least 1 month) and non-steroidal anti-inflammatory drugs ( $\geq 2$ weeks) were permitted.

Key exclusion criteria were failure of more than three agents for PsA (DMARDs or biologics) or more 
than one tumour necrosis factor blocker. Patients were also excluded if they had a history of or current (1) inflammatory, rheumatic or autoimmune joint disease other than PsA; (2) erythrodermic, guttate or generalised pustular psoriasis; (3) were functional class IV, defined by the American College of Rheumatology (ACR) Classification of Functional Status in Rheumatoid Arthritis; (4) had used phototherapy or DMARDs other than methotrexate, leflunomide or sulfasalazine within 4 weeks of randomisation; (5) had used adalimumab, etanercept, golimumab, infliximab, certolizumab pegol or tocilizumab within 12 weeks of randomisation or alefacept or ustekinumab within 24 weeks of randomisation; or (6) had prior treatment with apremilast. Topical therapy for psoriasis within 2 weeks of randomisation was not permitted. Patients with active tuberculosis or a history of incompletely treated tuberculosis could not participate.

\section{Study design}

PALACE 1, a phase 3, randomised, placebo-controlled study, was conducted at 83 sites in 13 countries beginning 2 June 2010 (clinical trial registration NCT01172938, ClinicalTrials.gov). Patients were randomised $(1: 1: 1)$ to placebo, apremilast $20 \mathrm{mg}$ twice a day (BID) or apremilast $30 \mathrm{mg}$ BID stratified by baseline DMARD use (yes/no). Apremilast was dose-titrated over the first week of treatment $(10 \mathrm{mg}$ on the first day, with increases of $10 \mathrm{mg} /$ day until the target dose was reached). Study visits were conducted at weeks 4, 16 and 24 for safety and weeks 16 and 24 for efficacy assessments. Patients whose swollen and tender joint counts had not improved by $\geq 20 \%$ were considered nonresponders at week 16 and were required to enter the protocoldefined early escape. Patients receiving placebo were re-randomised $(1: 1)$ to apremilast $20 \mathrm{mg}$ BID or $30 \mathrm{mg}$ BID, while those on apremilast remained on their initial apremilast dose. Treatment was continued through week 24 , at which time all remaining placebo patients were re-randomised (1:1) to apremilast $20 \mathrm{mg}$ BID or $30 \mathrm{mg}$ BID. Subsequently, all patients entered the 28 -week randomised, double-blind, active-treatment phase until week 52. Individual patient treatment assignments remained blinded to the investigators until final data analyses were completed after week 52. The active-treatment, long-term safety phase started at week 52, and patients are continuing their assigned treatment up to a total of 5 years. This report describes outcomes from the 24 -week placebo-controlled phase.

\section{Efficacy assessments}

The primary efficacy endpoint was the proportion of patients meeting 20\% improvement in modified ACR response criteria (ACR20) ${ }^{9}$ at week 16 . The key secondary endpoint was change from baseline in Health Assessment Questionnaire-Disability Index (HAQ-DI) at week 16. Additional efficacy outcome measures at week 24 included improvements in the signs and symptoms of PsA, physical function, enthesitis, dactylitis and psoriasis (see online supplementary table S1).

\section{Safety assessments}

Safety was evaluated at all scheduled clinic visits based on adverse events (AEs), vital signs, weight, physical examination and clinical laboratory investigations; a 12-lead electrocardiogram was obtained at screening, baseline and weeks 16 and 24. Safety assessments were also conducted in the event of early termination. There was no protocol requirement to stop study medication for an infection, no exclusions for vaccinations (including live virus) and no screening required for latent tuberculosis. AEs occurring after randomisation were classified using the Medical Dictionary for Drug Regulatory Activities Classification System.

\section{Statistical analysis}

Sample size estimations were based on the results of a phase 2 study of apremilast ${ }^{10}$; thus, it was estimated that 165 patients would be needed to provide $95 \%$ power to detect a $20 \%$ absolute difference in the ACR20 response between apremilast treatment and placebo using a two-group $\chi^{2}$ test with a 0.025 two-sided significance level. Efficacy was evaluated for the perprotocol population, which included all patients in the safety population (ie, randomised patients receiving at least one dose of study medication) who had at least one post-treatment ACR evaluation and no critical protocol violations. Protocol violations excluding patients from the per-protocol population were determined before unblinding of the 24-week database. The primary analysis of ACR20 response at week 16 was compared between apremilast treatment groups and placebo using a CochranMantel-Haenszel test adjusted for concomitant DMARD use at baseline. Patients who terminated the study early, before week 16 , or did not have sufficient data for assessment at week 16 were considered non-responders (non-responder imputation approach); last-observation-carried-forward methodology was used for the sensitivity analysis. Pairwise comparisons of each apremilast group versus placebo were performed. The Hochberg procedure was used to maintain type 1 error at the 0.05 significance level. Results were considered statistically significant if both the apremilast $20 \mathrm{mg}$ BID and $30 \mathrm{mg}$ BID versus placebo comparisons achieved $\mathrm{p}<0.05$, or one of the apremilast versus placebo comparisons achieved $\mathrm{p}<0.025$.

Analysis of covariance was used to compare the change in HAQ-DI score from baseline to week 16, with treatment and DMARD use as factors and baseline HAQ-DI score as a covariate. Categorical variables were analysed using the CochranMantel-Haenszel test, and continuous variables were analysed using the analysis of covariance model. Safety data were summarised descriptively for the safety population.

\section{RESULTS}

\section{Patients}

A total of 615 patients were screened and 504 were randomised and received at least one dose of study medication (figure 1). The pre-specified per-protocol efficacy population included 489 patients; $15(3.0 \%)$ were excluded for critical protocol violations, which included not having at least one post-baseline efficacy assessment that allowed for ACR20 calculation; one patient also had very low treatment compliance (41\%). In all, 444 (88.1\%) patients completed week 24.

Baseline demographics, disease characteristics and prior or concurrent therapy were comparable across treatment groups (table 1). In addition, these were generally consistent in the subgroup of biologic-experienced patients. As would be expected, relative to the overall population, patients with biologic experience tended to have a longer duration of both PsA and psoriasis, a greater proportion had baseline psoriasis body surface area $\geq 3 \%$, and a lower proportion were taking concomitant DMARDs at baseline. At baseline, 327 (64.9\%) patients were taking DMARDs, of whom $273(83.5 \%)$ were taking methotrexate; 119 (23.6\%) had prior biologic exposure, and 47 $(9.3 \%)$ were considered biologic therapeutic failures.

\section{Efficacy}

At week 16, significantly more patients receiving apremilast $20 \mathrm{mg}$ BID (51/163 (31.3\%); p=0.0140) and $30 \mathrm{mg}$ BID (64/ $161(39.8 \%) ; \mathrm{p}=0.0001)$ achieved an ACR20 response versus placebo (32/165 (19.4\%); figure 2A). The intent-to-treat 
Figure 1 Patient disposition through week 24.

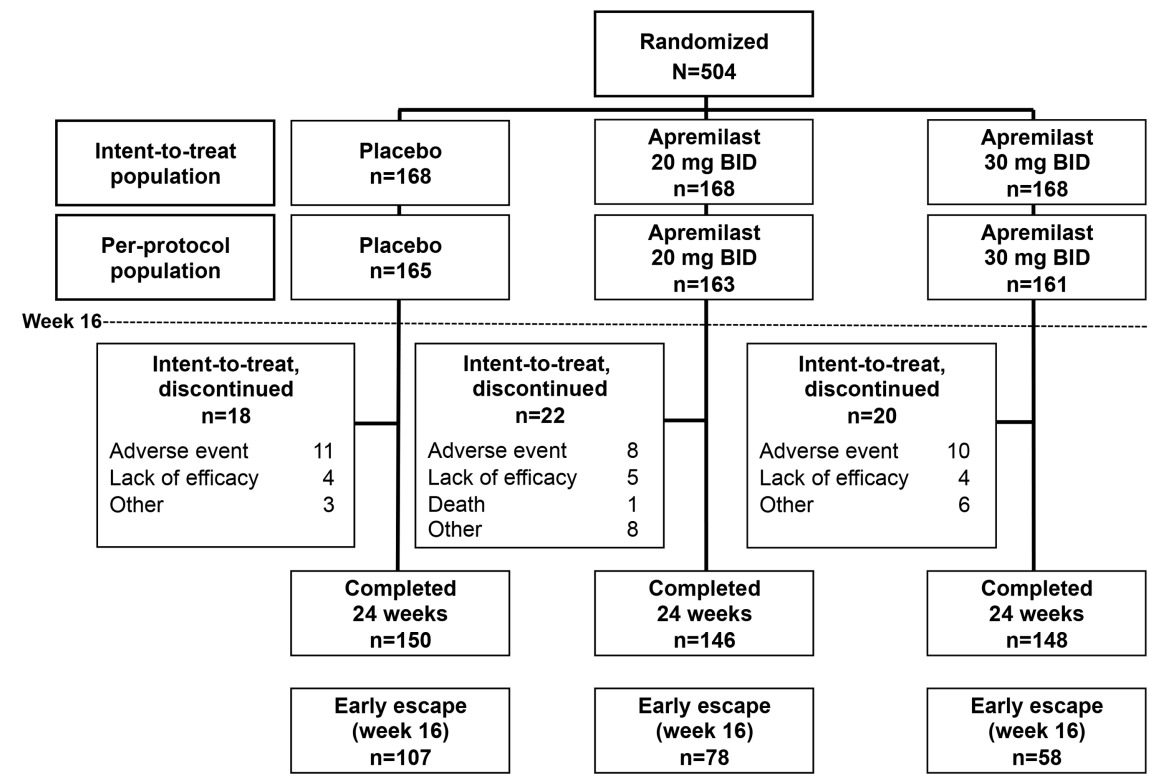

analysis, which included all patients who were randomised as specified in the protocol, demonstrated consistent results (placebo, 32/168 (19.0\%); apremilast $20 \mathrm{mg}$ BID, 51/168 (30.4\%), $\mathrm{p}=0.0166$; apremilast $30 \mathrm{mg}$ BID, 64/168 (38.1\%), $\mathrm{p}=0.0001)$. Efficacy was demonstrated across patients with varying treatment experience. Biologic-naïve patients generally experienced higher absolute ACR20 response rates compared with biologic-experienced patients and patients with a history of biologic failure (figure 2B). In general, a dose-related effect was observed with higher ACR20 response rates achieved in those receiving apremilast $30 \mathrm{mg}$ BID versus $20 \mathrm{mg}$ BID, although statistical comparison was not conducted.

\section{Secondary efficacy measures}

Week 16 values for baseline clinical characteristics are presented in online supplementary table S2. At week 16, apremilast was associated with significantly greater reductions (improvements) in HAQ-DI compared with placebo (key secondary endpoint). The mean (SE) changes from baseline were -0.09 (0.04) (placebo), -0.20 (0.04) (apremilast $20 \mathrm{mg} \mathrm{BID;} \mathrm{p}=0.0252$ vs placebo), and -0.25 (0.04) (apremilast $30 \mathrm{mg}$ BID; $\mathrm{p}=0.0015$ vs placebo). The intent-to-treat analysis demonstrated consistent results (placebo: -0.09 (0.04); apremilast $20 \mathrm{mg}$ BID: -0.20 (0.04) ( $\mathrm{p}=0.0252)$; apremilast $30 \mathrm{mg}$ BID: $-0.24 \quad(0.04)$ $(p=0.0017))$. At week 16 , a significantly greater proportion of patients receiving apremilast $30 \mathrm{mg}$ BID achieved minimal clinically important differences (MCID) of $\geq 0.13$ and $\geq 0.30$ on the HAQ-DI compared with placebo; differences between apremilast $20 \mathrm{mg}$ BID and placebo did not reach statistical significance. MCID $\geq 0.13$ was achieved by $38.8 \%$ (placebo), $44.8 \%$ (apremilast $20 \mathrm{mg} \mathrm{BID}$ ), and 50.3\% (apremilast $30 \mathrm{mg} \mathrm{BID;} \mathrm{p}=0.0334$ vs placebo) of patients. MCID $\geq 0.30$ was achieved by $27.3 \%$ (placebo), 33.7\% (apremilast $20 \mathrm{mg}$ BID) and 39.8\% (apremilast $30 \mathrm{mg}$ BID, $\mathrm{p}=0.0149$ vs placebo) of patients.

Table 2 summarises the effects of apremilast on additional efficacy measures at week 24 . A significantly greater proportion of patients receiving apremilast $20 \mathrm{mg}$ BID and $30 \mathrm{mg}$ BID achieved ACR20, ACR50 and ACR70 versus placebo; these response rates were maintained in the active treatment groups. An ACR20 response of 45.3\% was observed at week 24 in patients treated with apremilast $30 \mathrm{mg}$ BID independent of their response at week 16. A statistically significant improvement in physical function was observed with apremilast, as measured by changes from baseline in HAQ-DI score and the 36-Item Short-Form Health Survey v2 Physical Functioning domain score. Significant improvements in most ACR component scores, particularly swollen and tender joint counts and patient assessment of pain, were also observed (table 2).

In patients with baseline enthesitis, the mean change from baseline in the Maastricht Ankylosing Spondylitis Enthesitis Score (MASES) was significantly higher for apremilast $30 \mathrm{mg}$ BID versus placebo $(p=0.0334)$ (table 2$)$, and significantly greater proportions of patients receiving apremilast $20 \mathrm{mg}$ BID $(32 / 100$ (32.0\%); $\mathrm{p}=0.0037)$ and $30 \mathrm{mg}$ BID (36/107 (33.6\%); $\mathrm{p}=0.0013)$ achieved a MASES score of 0 at week 24 versus placebo (14/97 (14.4\%)). In patients with baseline dactylitis, mean change from baseline in dactylitis severity score was higher with apremilast versus placebo and resulted in greater proportions of patients with dactylitis scores achieving 0 at week 24 (apremilast $20 \mathrm{mg}$ BID: 29/57 (50.9\%); apremilast $30 \mathrm{mg}$ BID: $31 / 65(47.7 \%))$ versus placebo $(27 / 66(40.9 \%))$. However, these differences did not reach statistical significance at week 24 .

In patients with baseline psoriasis affecting $\geq 3 \%$ of the body surface area, significantly greater proportions of patients receiving either dose of apremilast achieved at least 50\% reduction from baseline Psoriasis Area and Severity Index ${ }^{11}$ (PASI-50) score (apremilast $20 \mathrm{mg}$ BID: 25/74 (33.8\%), p=0.0439; apremilast $30 \mathrm{mg}$ BID: 41/81 (50.6\%), $\mathrm{p}=0.0001)$ and PASI-75 score $(13 / 74$ (17.6\%), $\mathrm{p}=0.0180 ; 17 / 81$ (21.0\%), $\mathrm{p}=0.0040)$ versus placebo (PASI-50: 12/65 (18.5\%); PASI-75: 3/65 (4.6\%)).

\section{Safety}

During the 24-week placebo-controlled phase, AEs occurring in $\geq 5 \%$ of any treatment group included diarrhoea, nausea, headache and upper respiratory tract infection (table 3). Most AEs were mild to moderate in severity, and discontinuations due to AEs were comparable across groups (placebo: 8/168 (4.8\%); apremilast $20 \mathrm{mg}$ BID: 10/168 (6.0\%); apremilast $30 \mathrm{mg}$ BID: $12 / 168(7.1 \%))$. Gastrointestinal AEs were predominantly mild or moderate in severity, presented early, were self-limited and did not recur. Antidiarrhoeal use was low; 16 patients took antidiarrhoeal medication during the study, with 11 of these 
Table 1 Baseline demographics and clinical characteristics: intent-to-treat population $\left(\mathrm{N}=504^{*}\right)$

\begin{tabular}{|c|c|c|c|}
\hline & \multirow[b]{2}{*}{$\begin{array}{l}\text { Placebo } \\
n=168\end{array}$} & \multicolumn{2}{|l|}{ Apremilast } \\
\hline & & $\begin{array}{l}20 \mathrm{mg} \text { BID } \\
\mathrm{n}=168\end{array}$ & $\begin{array}{l}30 \mathrm{mg} \text { BID } \\
\mathrm{n}=168\end{array}$ \\
\hline Age, mean (SD), years & $51.1(12.1)$ & $48.7(11.0)$ & $51.4(11.7)$ \\
\hline Age $\geq 65$ years, $n$ & $19(11.3 \%)$ & $11(6.5 \%)$ & $22(13.1 \%)$ \\
\hline Female, $n$ & $80(47.6 \%)$ & $83(49.4 \%)$ & $92(54.8 \%)$ \\
\hline \multicolumn{4}{|l|}{ Race, $n$} \\
\hline White & $153(91.1 \%)$ & $150(89.3 \%)$ & $152(90.5 \%)$ \\
\hline Asian & $8(4.8 \%)$ & $8(4.8 \%)$ & $8(4.8 \%)$ \\
\hline Black & $0(0.0 \%)$ & $2(1.2 \%)$ & $0(0.0 \%)$ \\
\hline Other & $7(4.2 \%)$ & $8(4.8 \%)$ & $8(4.8 \%)$ \\
\hline \multicolumn{4}{|l|}{ Region, $\mathrm{n}$} \\
\hline North America & $81(48.2 \%)$ & $73(43.5 \%)$ & $69(41.1 \%)$ \\
\hline Europe & $39(23.2 \%)$ & $41(24.4 \%)$ & $42(25.0 \%)$ \\
\hline Rest of world & $48(28.6 \%)$ & $54(32.1 \%)$ & $57(33.9 \%)$ \\
\hline Weight, mean (SD), kg & $89.8(22.4)$ & $88.8(21.1)$ & $87.1(19.6)$ \\
\hline Body mass index, mean (SD), $\mathrm{kg} / \mathrm{m}^{2}$ & $31.1(6.6)$ & $30.9(7.3)$ & $30.6(5.9)$ \\
\hline Duration of psoriatic arthritis, mean (SD), years & $7.3(7.1)$ & $7.2(6.8)$ & $8.1(8.1)$ \\
\hline Swollen joint count (0-76), mean (SD) & $12.8(8.8)$ & $12.5(9.5)$ & $12.8(7.8)$ \\
\hline Tender joint count (0-78), mean (SD) & $23.3(15.2)$ & $22.2(15.9)$ & $23.1(14.5)$ \\
\hline HAQ-DI (0-3), mean (SD) & $1.2(0.6)$ & $1.2(0.6)$ & $1.2(0.6)$ \\
\hline Patient Global Assessment (0-100 mm VAS), mean (SD) & $58.8(22.3)$ & $55.3(23.7)$ & $55.9(21.5)$ \\
\hline Physician Global Assessment (0-100 mm VAS), mean (SD) & $55.2(20.3)$ & $54.1(21.8)$ & $55.7(19.2)$ \\
\hline CRP (mg/dL, normal range $<0.5$ ), mean (SD) & $1.1(1.436)$ & $0.90(1.409)$ & $0.84(1.024)$ \\
\hline Patient assessment of pain (0-100 mm VAS), mean (SD) & $61.2(20.2)$ & $54.9(22.9)$ & $57.9(20.2)$ \\
\hline SF-36v2 PF score, mean (SD) & $33.8(10.6)$ & $35.1(10.7)$ & $33.0(10.2)$ \\
\hline DAS-28 (CRP), mean (SD) & $4.9(1.0)$ & $4.8(1.1)$ & $4.9(1.0)$ \\
\hline CDAI (0-76), mean (SD) & $29.7(12.0)$ & $28.4(13.1)$ & $29.4(11.5)$ \\
\hline Duration of psoriasis, mean (SD), years & $15.7(13.0)$ & $15.5(11.9)$ & $16.5(12.3)$ \\
\hline Psoriasis involvement of body surface area $\geq 3 \%, n$ & $68(40.5 \%)$ & $77(45.8 \%)$ & $82(48.8 \%)$ \\
\hline PASI score $(0-72), \dagger$ mean (SD) & $9.1(9.5)$ & $7.4(8.7)$ & $9.2(9.7)$ \\
\hline Presence of enthesitis, $n$ & $98(58.3 \%)$ & $103(61.3 \%)$ & $114(67.9 \%)$ \\
\hline MASES $(0-13), \ddagger$ mean (SD) & $5.4(3.5)$ & $5.0(3.3)$ & $4.4(3.1)$ \\
\hline Presence of dactylitis, $n$ & $68(40.5 \%)$ & $59(35.1 \%)$ & $68(40.5 \%)$ \\
\hline Dactylitis severity score $(0-20), \S$ mean (SD) & $3.3(3.3)$ & $4.1(4.2)$ & $2.9(2.4)$ \\
\hline Prior use of DMARDs (biologic-naïve), $n$ & $120(71.4 \%)$ & $129(76.8 \%)$ & $124(73.8 \%)$ \\
\hline Prior use of biologics, $n$ & $41(24.4 \%)$ & $37(22.0 \%)$ & $41(24.4 \%)$ \\
\hline Prior biologic failures, $\mathrm{n}$ & $19(11.3 \%)$ & $14(8.3 \%)$ & $14(8.3 \%)$ \\
\hline Baseline DMARD use, $\mathrm{n}$ & $110(65.5 \%)$ & $111(66.1 \%)$ & $106(63.1 \%)$ \\
\hline Methotrexate (mean dose, $16.6 \mathrm{mg} /$ week) & $90(53.6 \%)$ & $95(56.5 \%)$ & $88(52.4 \%)$ \\
\hline Leflunomide (mean dose, 17.2 mg/day) & $11(6.5 \%)$ & $10(6.0 \%)$ & $9(5.4 \%)$ \\
\hline Sulfasalazine (mean dose, $2.3 \mathrm{~g} /$ day) & $18(10.7 \%)$ & $16(9.5 \%)$ & $20(11.9 \%)$ \\
\hline Baseline corticosteroids, $1 \mathrm{n}$ (mean dose, $6.1 \mathrm{mg} / \mathrm{day}$ ) & $12(7.1 \%)$ & $25(14.9 \%)$ & $16(9.5 \%)$ \\
\hline Baseline use of NSAIDs, $n$ & $118(70.2 \%)$ & $123(73.2 \%)$ & $120(71.4 \%)$ \\
\hline
\end{tabular}

\footnotetext{
*The $\mathrm{n}$ reflects the number of randomised patients; actual number of patients available for each endpoint may vary.

tExamined among patients who had body surface area $\geq 3 \%$ affected at baseline.

¥Examined among patients who had enthesitis at baseline.

$\S$ Examined among patients who had dactylitis at baseline.

१Prednisone $\leq 10 \mathrm{mg} / \mathrm{day}$ (or equivalent).

CDAI, Clinical Disease Activity Index; CRP, C-reactive protein; DAS-28, 28-joint Disease Activity Score; DMARDs, disease-modifying antirheumatic drugs; HAQ-DI, Health Assessment Questionnaire-Disability Index; MASES, Maastricht Ankylosing Spondylitis Enthesitis Score; NSAIDs, non-steroidal anti-inflammatory drugs; PASI, Psoriasis Area and Severity Index; SF-36V2 PF, 36-Item Short-Form Health Survey v2 Physical Functioning domain; VAS, visual analogue scale.
}

patients taking medication while experiencing diarrhoea as an AE. Hence, a low rate of discontinuation was associated with gastrointestinal AEs (placebo: 4/168 (2.4\%); apremilast $20 \mathrm{mg}$ BID: $3 / 168$ (1.8\%); apremilast $30 \mathrm{mg}$ BID: $7 / 168$ (4.2\%)). Severe nausea was reported by one patient in each apremilast group, and severe diarrhoea was reported by one patient each in the placebo and apremilast $20 \mathrm{mg}$ BID groups. In patients receiving apremilast, median onset of diarrhoea (56 events in 51 patients) and nausea (59 events in 47 patients) was 9 and 10 days, respectively, and the median duration was 29.5 and 17 days. Of note, the first study visit occurred at week 4.

Serious AEs were low and comparable across treatment groups. Among these were four serious infections, two of which occurred with placebo (cellulitis and bacterial wound infection) and two of which occurred with apremilast $30 \mathrm{mg}$ BID (gastrointestinal clostridial infection and pneumonia). All patients with 
A Overall Population (Primary Endpoint)

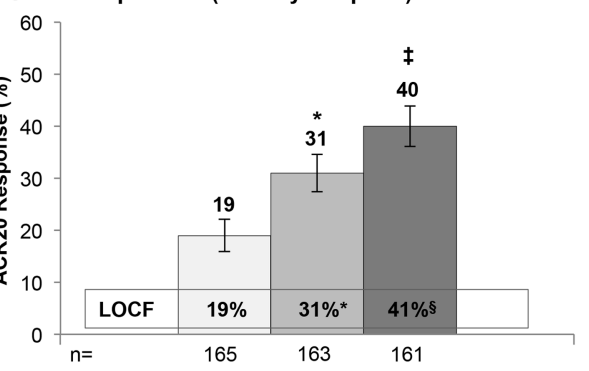

B Biologic Experience

$$
60
$$

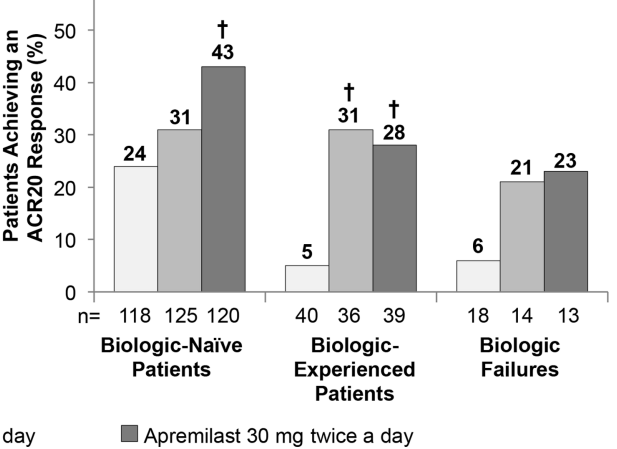

Figure 2 Proportion of patients achieving 20\% improvement in modified American College of Rheumatology (ACR20) response criteria at week 16. (A) The proportion of patients achieving an ACR20 response at week 16 (primary efficacy endpoint). (B) The proportion of patients achieving an ACR20 response by biologic experience. The per-protocol population $(n=489)$ was analysed using non-responder imputation for missing data; last-observation-carried-forward (LOCF) methodology was used for sensitivity analyses. Error bars represent $S E$. ${ }^{*} p<0.02 ; \quad t p<0.007 ; \neq p<0.001$.

serious infections recovered after standard courses of antibiotic treatment and continued the study. Other serious events included two myocardial infarctions (placebo and apremilast $20 \mathrm{mg}$ BID) and two solid tumour malignancies (placebo: prostate cancer; apremilast $30 \mathrm{mg}$ BID: breast cancer). One death occurred on day 73 in a 52-year-old woman receiving apremilast
$20 \mathrm{mg}$ BID plus methotrexate; the primary cause of death was multiorgan failure secondary to pre-existing vitamin B12 deficiency and was considered unrelated to study medication by the investigator. No cases of active tuberculosis (new or reactivation, despite no latent tuberculosis screening requirements), lymphoma or vasculitis were reported.

Table 2 Secondary outcomes at week 24: per-protocol population $(n=489 *)$

\begin{tabular}{|c|c|c|c|c|c|}
\hline & \multirow[b]{2}{*}{$\begin{array}{l}\text { Placebo } \\
n=165\end{array}$} & \multicolumn{4}{|l|}{ Apremilast } \\
\hline & & $\begin{array}{l}20 \mathrm{mg} \text { BID } \\
\mathrm{n}=163\end{array}$ & $\begin{array}{l}p \text { Value vs } \\
\text { placebo }\end{array}$ & $\begin{array}{l}30 \mathrm{mg} \text { BID } \\
\mathrm{n}=161\end{array}$ & $\mathrm{p}$ Value vs placebo \\
\hline ACR20, nt & $22(13.3 \%)$ & $43(26.4 \%)$ & 0.0032 & $59(36.6 \%)$ & $<0.0001$ \\
\hline ACR50, nt & $7(4.2 \%)$ & $24(14.7 \%)$ & 0.0013 & $32(19.9 \%)$ & $<0.0001$ \\
\hline ACR70, nt & $1(0.6 \%)$ & $9(5.5 \%)$ & 0.0102 & $17(10.6 \%)$ & 0.0001 \\
\hline HAQ-DI (0-3), LS mean change (SE) & $-0.08(0.04)$ & $-0.21(0.04)$ & 0.0092 & $-0.26(0.04)$ & 0.0004 \\
\hline SF-36v2 PF score, LS mean change (SE) $\ddagger$ & $1.5(0.67)$ & $3.5(0.68)$ & 0.0295 & $5.1(0.67)$ & 0.0001 \\
\hline EULAR good/moderate response, $n$ & $27(16.4 \%)$ & $51(31.3 \%)$ & 0.0016 & $71(44.1 \%)$ & $<0.0001$ \\
\hline DAS-28 (CRP), LS mean change (SE) & $-0.20(0.09)$ & $-0.66(0.09)$ & 0.0002 & $-0.91(0.09)$ & $<0.0001$ \\
\hline DAS-28 (CRP) $<2.6, n$ & $4(2.4 \%)$ & $19(11.7 \%)$ & 0.0011 & $30(18.6 \%)$ & $<0.0001$ \\
\hline CDAI (0-76), LS mean change (SE) & $-3.1(0.97)$ & $-7.6(0.96)$ & 0.0010 & $-9.6(0.95)$ & $<0.0001$ \\
\hline Patient assessment of pain (0-100 mm VAS), LS mean change (SE) & $-4.1(1.8)$ & $-11.3(1.8)$ & 0.0045 & $-14.8(1.8)$ & $<0.0001$ \\
\hline Swollen joint count (0-76), LS mean change (SE) & $-1.4(0.63)$ & $-4.1(0.63)$ & 0.0023 & $-5.1(0.63)$ & $<0.0001$ \\
\hline Tender joint count (0-78), LS mean change (SE) & $-0.91(1.01)$ & $-5.0(1.0)$ & 0.0035 & $-7.8(1.0)$ & $<0.0001$ \\
\hline Patient Global Assessment (0-100 mm VAS), LS mean change (SE) & $-2.1(1.9)$ & $-8.0(1.9)$ & 0.0285 & $-12.1(1.9)$ & 0.0002 \\
\hline Physician Global Assessment (0-100 mm VAS), LS mean change (SE) & $-6.7(1.9)$ & $-14.4(1.9)$ & 0.0040 & $-19.1(1.9)$ & $<0.0001$ \\
\hline CRP (mg/dL, normal range $<0.5$ ), LS mean change (SE) & $0.17(0.09)$ & $-0.02(0.09)$ & 0.1321 & $-0.05(0.09)$ & 0.0713 \\
\hline MASES (0-13),§ LS mean change (SE) & $-0.8(0.31)$ & $-1.6(0.30)$ & 0.0678 & $-1.7(0.29)$ & 0.0334 \\
\hline Dactylitis severity score (0-20), I LS mean change (SE) & $-1.3(0.27)$ & $-2.0(0.30)$ & 0.0710 & $-1.8(0.27)$ & 0.1753 \\
\hline PASI-50, $n^{* *}$ & $12(18.5 \%)$ & $25(33.8 \%)$ & 0.0439 & 41 (50.6\%) & 0.0001 \\
\hline PASI-75, $n^{* *}$ & $3(4.6 \%)$ & $13(17.6 \%)$ & 0.0180 & $17(21.0 \%)$ & 0.0040 \\
\hline
\end{tabular}

Imputation methods included non-responder imputation for categorical endpoints that involve joint counts and last observation carried forward for all continuous endpoints and categorical endpoints that do not involve joint counts.

*The $\mathrm{n}$ reflects the number of randomised patients in the per-protocol population; actual number of patients available for each endpoint may vary.

tPatients who escaped early, discontinued early or did not have sufficient data for ACR response determination were counted as non-responders.

\#Increase in score from baseline indicates improvement.

§Examined among patients who had enthesitis at baseline and $\geq 1$ post-baseline value at or prior to week 24 (placebo: $n=96$; apremilast $20 \mathrm{mg}$ BID: $n=100 ;$ apremilast $30 \mathrm{mg}$ BID: $\mathrm{n}=107)$.

IExamined among patients who had dactylitis at baseline and $\geq 1$ post-baseline value at or prior to week 24; each digit on the patient's hand and feet was assessed for presence (score $=1$ ) or absence $($ score $=0$ ) of dactylitis. The dactylitis score was the sum of the individual assessments for all 20 digits (placebo: $n=64 ;$ apremilast $20 \mathrm{mg} B$ BID: $n=56$; apremilast $30 \mathrm{mg}$ BID: $\mathrm{n}=65$ ).

**Examined among patients who had body surface area $\geq 3 \%$ at baseline (placebo: $n=65$; apremilast $20 \mathrm{mg}$ BID: $n=74$; apremilast $30 \mathrm{mg}$ BID: $n=81$ ).

ACR20/50/70, 20\%/50\%/70\% improvement in modified American College of Rheumatology response criteria; CDAl, Clinical Disease Activity Index; CRP, C-reactive protein; DAS-28

(CRP), 28-joint Disease Activity Score (using CRP as acute-phase reactant); EULAR, European League Against Rheumatism; HAQ-DI, Health Assessment Questionnaire-Disability Index;

LS, least-squares; MASES, Maastricht Ankylosing Spondylitis Enthesitis Score; PASI-50/75, 50\%/75\% reduction from baseline Psoriasis Area and Severity Index score; SF-36v2 PF, 36-Item

Short-Form Health Survey Physical Functioning domain; VAS, visual analogue scale. 
Table 3 Adverse events and laboratory abnormalities during the placebo-controlled phase (weeks 0-24)*

\begin{tabular}{|c|c|c|c|}
\hline \multirow[b]{2}{*}{ Patients } & \multirow[b]{2}{*}{$\begin{array}{l}\text { Placebo } \\
n=168\end{array}$} & \multicolumn{2}{|l|}{ Apremilast } \\
\hline & & $\begin{array}{l}20 \mathrm{mg} \text { BID } \\
n=168\end{array}$ & $\begin{array}{l}30 \mathrm{mg} \text { BID } \\
\mathrm{n}=168\end{array}$ \\
\hline \multicolumn{4}{|l|}{ Overview of adverse events, $n$} \\
\hline Any adverse event & $81(48.2 \%)$ & $101(60.1 \%)$ & $103(61.3 \%)$ \\
\hline Any severe adverse event & $6(3.6 \%)$ & $8(4.8 \%)$ & $11(6.5 \%)$ \\
\hline Any serious adverse event & $7(4.2 \%)$ & $8(4.8 \%)$ & $9(5.4 \%)$ \\
\hline Any adverse event leading to drug withdrawal & $8(4.8 \%)$ & $10(6.0 \%)$ & $12(7.1 \%)$ \\
\hline \multicolumn{4}{|c|}{ Adverse events reported by $\geq 5 \%$ of patients in any treatment group, $n$} \\
\hline Diarrhoea & $4(2.4 \%)$ & $19(11.3 \%)$ & $32(19.0 \%)$ \\
\hline Nausea & $11(6.5 \%)$ & $16(9.5 \%)$ & $31(18.5 \%)$ \\
\hline Headache & $8(4.8 \%)$ & $17(10.1 \%)$ & $18(10.7 \%)$ \\
\hline Upper respiratory tract infection & $6(3.6 \%)$ & $10(6.0 \%)$ & $7(4.2 \%)$ \\
\hline \multicolumn{4}{|c|}{ Adverse events leading to discontinuation in $>1$ patient in any treatment group, $n$} \\
\hline Diarrhoea & $3(1.8 \%)$ & $0(0.0 \%)$ & $4(2.4 \%)$ \\
\hline Nausea & $2(1.2 \%)$ & $2(1.2 \%)$ & $3(1.8 \%)$ \\
\hline Migraine & $0(0.0 \%)$ & $1(0.6 \%)$ & $2(1.2 \%)$ \\
\hline \multicolumn{4}{|c|}{ Patients with select laboratory value shifts from normal to $>$ the upper limit of normal, nt } \\
\hline Alanine transaminase, $\mathrm{U} / \mathrm{L}$ & $20 / 150(13.3 \%)$ & $12 / 146(8.2 \%)$ & $12 / 155(7.7 \%)$ \\
\hline Creatinine, $\mu \mathrm{mol} / \mathrm{L}$ & $3 / 159(1.9 \%)$ & $7 / 151(4.6 \%)$ & $10 / 158(6.3 \%)$ \\
\hline \multicolumn{4}{|c|}{ Patients with select laboratory value shifts from normal to $<$ the lower limit of normal, nt } \\
\hline Leukocytes, $10^{9} / \mathrm{L}$ & $1 / 155(0.6 \%)$ & $4 / 155(2.6 \%)$ & $2 / 159(1.3 \%)$ \\
\hline Neutrophils, $10^{9} / \mathrm{L}$ & $2 / 146(1.4 \%)$ & $2 / 145(1.4 \%)$ & $5 / 151(3.3 \%)$ \\
\hline Platelets, $10^{9} / \mathrm{L}$ & $0 / 146(0.0 \%)$ & $0 / 142(0.0 \%)$ & $1 / 151(0.7 \%)$ \\
\hline Haemoglobin, g/dL & $8 / 148(5.4 \%)$ & $7 / 149(4.7 \%)$ & $14 / 153(9.2 \%)$ \\
\hline
\end{tabular}

Clinically meaningful laboratory abnormalities were infrequent, self-limited, and comparable between apremilast treatment arms and placebo. Observed weight loss was treatment-related, with $<2 \%$ of patients with weight-related AEs. Mean (SD) weight change from baseline up to week 24 was 0.19 (2.6) $\mathrm{kg}$ with placebo $(\mathrm{n}=167),-1.29$ (3.4) $\mathrm{kg}$ with apremilast $20 \mathrm{mg}$ BID $(\mathrm{n}=166)$ and $-0.97(2.8) \mathrm{kg}$ with apremilast $30 \mathrm{mg}$ BID $(\mathrm{n}=168)$.

\section{DISCUSSION}

PsA is a chronic immune disease associated with significant morbidity. Traditional DMARDs remain the mainstay of PsA treatment. However, currently available DMARDs have demonstrated variable efficacy in treating all of the rheumatologic and dermatologic manifestations of PsA. The biologic agents, particularly tumour necrosis factor blockers, have demonstrated substantial responses for many, but not all, patients. All available agents have some potential safety concerns. Thus, there is an unmet medical need for novel therapeutic agents that address the varied clinical manifestations of PsA and offer a favourable benefit/risk profile.

This study presents the first phase 3 data demonstrating the efficacy of apremilast, an oral phosphodiesterase 4 inhibitor, in patients with PsA and prior experience with traditional DMARDs and/or biologic therapies, including treatment failures. The majority of patients $(>74.0 \%)$ had received prior treatment with one or more traditional DMARDs; $64.9 \%$ were on at least one DMARD at baseline, most commonly methotrexate. Of note, $23.6 \%$ of the patients had previous biologic exposure and $9.3 \%$ were considered biologic therapeutic failures. The enrolled patients were representative of a population with active, long-standing disease and a history of treatment experience. C-reactive protein (CRP) levels at baseline were lower than other PsA studies that, unlike the current study, had enrolment requirement criteria for $\mathrm{CRP}^{12}{ }^{13}$ and, as expected, may be lower than patient populations with rheumatoid arthritis, as PsA is generally associated with lower CRP levels compared with rheumatoid arthritis. ${ }^{14}$

Both doses of apremilast significantly improved the signs and symptoms of PsA at week 16. Efficacy was observed with apremilast regardless of prior biologic experience or concomitant DMARD use, with a higher absolute rate of ACR20 response in biologic-naïve patients. Apremilast demonstrated statistically significant improvements in physical function, as measured by the HAQ-DI score at week 16. Continued dosing through week 24 maintained or further improved the signs and symptoms of PsA and physical function. Results based on additional efficacy measures, including ACR50, ACR70, swollen and tender joint counts, 36-item Short-Form Health Survey v2 Physical Functioning domain and PASI-75 score, were consistent with the positive ACR20 response rates and change in HAQ-DI score. A significantly greater proportion of patients receiving apremilast $20 \mathrm{mg} \mathrm{BID}$ and $30 \mathrm{mg}$ BID achieved DAS-28 (CRP) remission $(<2.6)$ compared with placebo. Across most efficacy measures, response rates and improvements were greater with apremilast $30 \mathrm{mg}$ BID versus apremilast $20 \mathrm{mg}$ BID, although the study was not designed to make formal efficacy comparisons.

Apremilast at doses of $20 \mathrm{mg}$ BID and $30 \mathrm{mg}$ BID was generally well tolerated over 24 weeks. The most common AEs were largely gastrointestinal, including diarrhoea and nausea; most of these occurred in the first month of treatment, were 
predominantly mild or moderate in severity, and did not lead to discontinuation. Two serious infections (gastrointestinal clostridial infection, pneumonia) occurred in the apremilast treatment groups; each resolved with appropriate antibiotic treatment, and patients continued apremilast without interruption. No imbalance was observed between placebo and apremilast in terms of major adverse cardiac events, serious infections including systemic opportunistic infections or malignancies, including lymphoma. No cases of active tuberculosis or vasculitis were reported. Apremilast use did not result in clinically meaningful laboratory abnormalities, suggesting that routine laboratory monitoring may not be required when using apremilast.

The study was limited in duration, and results from the ongoing long-term phase are anticipated. This is the first study in the PALACE apremilast clinical trial programme, which comprises four phase 3 randomised, placebo-controlled trials with long-term, open-label extensions for up to 5 years. The programme is one of the largest to date in PsA patients who have prior DMARD and/or biologic experience. The significant efficacy observed in PALACE 1 , across multiple domains of PsA, along with the safety and tolerability profile, and potential lack of requirement for laboratory monitoring, suggest apremilast may become an important addition to the current PsA treatment armamentarium.

\section{CONCLUSION}

These findings demonstrate that apremilast is effective for the treatment of active PsA across a diverse group of patients with prior treatment experience, alone or in combination with traditional DMARDs. Furthermore, apremilast was well tolerated in the majority of patients and demonstrated an acceptable safety profile. These results confirm the therapeutic potential of apremilast BID for treatment of patients with PsA.

\author{
Author affiliations \\ ${ }^{1}$ Division of Rheumatology, Allergy and Immunology, Department of Medicine, \\ University of California at San Diego, La Jolla, California, USA \\ ${ }^{2}$ Swedish Medical Center and University of Washington School of Medicine, Seattle, \\ Washington, USA \\ ${ }^{3}$ Hospital Clinico Universitario, Santiago, Spain \\ ${ }^{4}$ University of Sheffield, Sheffield, UK \\ ${ }^{5}$ Schön Klinik Hamburg Eilbek, Klinik für Rheumatologie, Hamburg, Germany \\ ${ }^{6}$ Division of Health Care \& Outcomes Research, Toronto Western Research Institute, \\ Toronto, Ontario, Canada \\ ${ }^{7}$ University of Orléans, Orléans, France \\ ${ }^{8}$ Monash University, CabriniHealth, Melbourne, Australia \\ ${ }^{9}$ Celgene Corporation, Summit, New Jersey, USA \\ ${ }^{10}$ Department of Internal Medicine 3, University of Erlangen-Nuremberg, Erlangen, \\ Germany
}

Acknowledgements The authors thank Maricar Postaski (data management, clinical operations), Peter Maes, Colleen Newman, Rosemary Petric, Yufang Lu, and Adele Vessey (clinical research), Elisabeth Kurkimilis (clinical operations), Andrew Wang (programming), and Xiaojiang Zhan (statistics) of Celgene Corporation for their contributions to the manuscript.

Contributors AK: study design, data acquisition, data analysis and manuscript drafting. PJM, JJG-R, AOA, JW, DDG, EL, SH and GS: study design, data acquisition and critical comments to manuscript drafting. $\mathrm{MH}, \mathrm{CCH}, \mathrm{DH}$ and RMS: study design, data acquisition, data analysis and critical comments to manuscript drafting. The authors, however, directed and are fully responsible for all content and editorial decisions for this manuscript.

Funding This study was sponsored by Celgene Corporation.

Competing interests $A K$ has provided expert advice to and/or received research grants from Abbott, Amgen, Astra-Zeneca, BMS, Celgene Corporation, Centocor-Janssen, Pfizer, Roche and UCB. PJM has received research grants and consultant fees from and served on the speaker's bureau for Abbott, Amgen, Biogen Idec, BMS, Genentech, Janssen, Eli Lilly, Pfizer and UCB, and received research grants and consultant fees from Celgene Corporation, Novartis and Roche. JJG-R has served on the advisory boards of BMS, Pfizer, Roche, Schering-Plough and UCB SA; received lecture fees from BMS, Roche, Schering-Plough and Wyeth; and received research grants from Roche and Schering-Plough. AOA and SH have no relevant competing interests to disclose. JW has received consulting fees from Abbott, Bristol-Myers Squibb, MSD, Pfizer and UCB. DDG has received grants and consulting fees from AbbVie, Amgen, BMS, Celgene Corporation, Janssen, Pfizer, Novartis and UCB. EL has received grants and research support from Novartis, Lilly, Servier, and Amgen and served on the speaker's bureau for Novartis and Eli Lilly. MH, CCH, DH and RMS are employees of Celgene Corporation. GS has received grants and research support from Celgene Corporation and consulting fees from Abbott, UCB and Roche. The authors received editorial support in the preparation of this manuscript from Peloton Advantage, LLC, and Jennifer Schwinn, RPh, funded by Celgene Corporation.

\section{Patient consent Obtained.}

Ethics approval The institutional review boards from each investigation site approved this study in accordance with the Declaration of Helsinki, the International Conference on Harmonization Guideline E6: Good Clinical Practice, and Title 21 of the Code of Federal Regulations, Part 50 Protection of Human Subjects and Part 56 Institutional Review Boards.

Provenance and peer review Not commissioned; externally peer reviewed.

Open Access This is an Open Access article distributed in accordance with the Creative Commons Attribution Non Commercial (CC BY-NC 3.0) license, which permits others to distribute, remix, adapt, build upon this work non-commercially, and license their derivative works on different terms, provided the original work is properly cited and the use is non-commercial. See: http://creativecommons.org/ licenses/by-nc/3.0/

\section{REFERENCES}

1 Gladman DD, Antoni C, Mease $\mathrm{P}$, et al. Psoriatic arthritis: epidemiology, clinical features, course, and outcome. Ann Rheum Dis 2005;64(Suppl 2):ii14-17.

2 Ash Z, Gaujoux-Viala C, Gossec L, et al. A systematic literature review of drug therapies for the treatment of psoriatic arthritis: current evidence and meta-analysis informing the EULAR recommendations for the management of psoriatic arthritis. Ann Rheum Dis 2012;71:319-26.

3 Gossec L, Smolen JS, Gaujoux-Viala C, et al. European League Against Rheumatism recommendations for the management of psoriatic arthritis with pharmacological therapies. Ann Rheum Dis 2012;71:4-12.

4 Kingsley GH, Kowalczyk A, Taylor $\mathrm{H}$, et al. A randomized placebo-controlled trial of methotrexate in psoriatic arthritis. Rheumatology (Oxford) 2012;51:1368-77.

5 Menter A, Korman NJ, Elmets CA, et al. Guidelines of care for the management of psoriasis and psoriatic arthritis: section 4. Guidelines of care for the management and treatment of psoriasis with traditional systemic agents. J Am Acad Dermatol 2009:61:451-85

6 Schafer P. Apremilast mechanism of action and application to psoriasis and psoriatic arthritis. Biochem Pharmacol 2012;83:1583-90.

7 Schafer PH, Parton A, Gandhi AK, et al. Apremilast, a CAMP phosphodiesterase-4 inhibitor, demonstrates anti-inflammatory activity in vitro and in a model of psoriasis. Br J Pharmacol 2010;159:842-55.

8 Taylor W, Gladman D, Helliwell P, et al. Classification criteria for psoriatic arthritis: development of new criteria from a large international study. Arthritis Rheum 2006;54:2665-73.

9 Felson DT, Anderson JJ, Boers M, et al. American College of Rheumatology. Preliminary definition of improvement in rheumatoid arthritis. Arthritis Rheum 1995;38:727-35.

10 Schett G, Wollenhaupt J, Papp K, et al. Oral apremilast in the treatment of active psoriatic arthritis: results of a multicenter, randomized, double-blind, placebocontrolled study. Arthritis Rheum 2012;64:3156-67.

11 Mease PJ. Measures of psoriatic arthritis: tender and swollen joint assessment, Psoriasis Area and Severity Index (PASI), Nail Psoriasis Severity Index (NAPSI), Modified Nail Psoriasis Severity Index (mNAPSI), Mander/Newcastle Enthesitis Index (MEI), Leeds Enthesitis Index (LEI), Spondyloarthritis Research Consortium of Canada (SPARCC), Maastricht Ankylosing Spondylitis Enthesis Score (MASES), Leeds Dactylitis Index (LDI), Patient Global for Psoriatic Arthritis, Dermatology Life Quality Index (DLQI), Psoriatic Arthritis Quality of Life (PsAQOL), Functional Assessment of Chronic Illness Therapy-Fatigue (FACIT-F), Psoriatic Arthritis Response Criteria (PsARC), Psoriatic Arthritis Joint Activity Index (PsAJAI), Disease Activity in Psoriatic Arthritis (DAPSA), and Composite Psoriatic Disease Activity Index (CPDAI). Arthritis Care Res (Hoboken) 2011;63(Suppl 11):S64-85

12 Mclnnes IB, Kavanaugh A, Gottlieb AB, et al. Efficacy and safety of ustekinumab in patients with active psoriatic arthritis: 1 year results of the phase 3 , multicentre, double-blind, placebo-controlled PSUMMIT 1 trial. Lancet 2013:382:780-9.

13 Kavanaugh A, McInnes I, Mease P, et al. Golimumab, a new human tumor necrosis factor alpha antibody, administered every four weeks as a subcutaneous injection in psoriatic arthritis: twenty-four-week efficacy and safety results of a randomized, placebo-controlled study. Arthritis Rheum 2009:60:976-86.

14 Lindqvist UR, Alenius GM, Husmark T, et al. The Swedish early psoriatic arthritis register-2-year followup: a comparison with early rheumatoid arthritis. J Rheumatol 2008;35:668-73. 